\title{
Sharpness of Tire Angle Determination Based on Point Cloud Dataset
}

\author{
Xinrong Lyu* \\ College of Information and Control Engineering, China University of Petroleum (East China), Qingdao 266580, China \\ ${ }^{*}$ Corresponding author
}

\begin{abstract}
In the tire production line, it is usually necessary to trim the blank tires to finished tires according to the sharpness of tire angle. If the determination of tire angle is made by mistakes, it will cause the tire to be a waste for choosing a wrong scraper. In order to determine the sharpness of tire angle accurately, point cloud acquisition camera was used to collect the data of tires in the production line. Based on the point cloud data of tires, a framework to detect the sharpness of tire angle was proposed. In the proposed framework, a locating method using image processing algorithms such as morphological operation, connected component labeling and corner point detection was used to locate the tire angle. A ridge detection algorithm including surface normal vector and principal curvature computation was used to determine the type of tire angle. A number of point cloud datasets of different tires experimental results show that the proposed framework has higher accuracy and efficiency to determine whether the tire angle is sharp or round.
\end{abstract}

Keywords_point cloud; sharpness; tire angle

\section{INTRODUCTION}

With the rapid development of 3D (three-dimensional) scanning technology and equipment, it becomes more and more easier to obtain the surface data of a certain object. The point cloud data obtained by 3D scanner usually contain 3D coordinates and color attributes of sample points. The point cloud data can be processed, and the point cloud model can be constructed, and the 3D molding can be realized quickly. The point cloud model, which is generated by a 3D laser scanner, reveals the terrain condition, shape, texture and spatial structure of the object, and realizes the virtual reshaping of space by accuracy to millimeter error. At present, feature extraction of 3D model has become a hot topic in computer vision and digital geometry processing technology, and it is widely used in industrial design, medicine, art and so on. Feature is an important part of geometric model. It plays an important role in the appearance, modeling and accurate expression of geometric models [1]. In view of the feature extraction of point cloud data, many researchers at home and abroad have made some related research work. In multi-scale feature extraction, Gumhold [2] used the eigenvalue of local neighborhood covariance matrix as a measure to calculate the weight of a point. Pauly [3] calculated the possibility that one point becomes a feature point under different scales by taking the size of the local neighborhood as the discrete scale parameter. In Pang's research [4], according to the local surface fitting polynomial of the point cloud model, the principal curvature and the main direction of each point in the point cloud model were calculated, and the potential valley ridge feature points were marked with the main principal curvature with a bigger absolute value. Then, the enhanced valley ridge points were obtained by projecting the obtained feature points to the main axis of the neighborhood points, and a smooth valley and ridge would be obtained by smoothing the valley ridge points finally. Mergot [5] used the Voronoi covariance measure to extract the feature of point cloud based on the effective calculation of point cloud curvature, sharp feature and normal direction. An[6] realized the feature recognition of the regular 3D point cloud data based on estimation of the geometric characteristics of discrete curves and discrete surfaces.

In the tire production line, it is necessary to determine the sharpness of the tire angle, seen in Figure I. According to the type of tire determined, an appropriate scraper will be selected to eliminate the part which does not meet the requirement of standard tire, such as the surplus part in the tires which has a sharp angle but the standard one should have a round angle. If the type of tires is identified not correctly, they will cause mistakes in the selection of scraper type, which will result in the loss of tire waste. In order to solve the sharpness of tire angle determination problem in the tire production line, a framework is proposed based on point cloud datasets of tires. Firstly, the locations of tire angle are determined based on their characteristics in the 2D cut image processing including morphological operation, connected component labeling and corner point detection. Then, according to the surface normal vector and the principal curvature, a ridge detection method is used to determine the sharpness of tire angle.

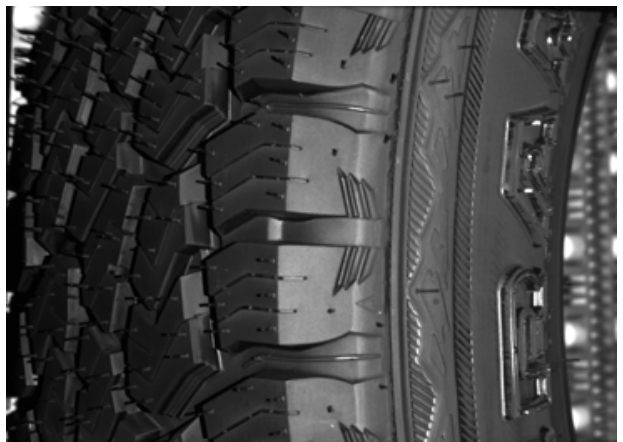

FIGURE I. (a)A tire with sharp angle. 


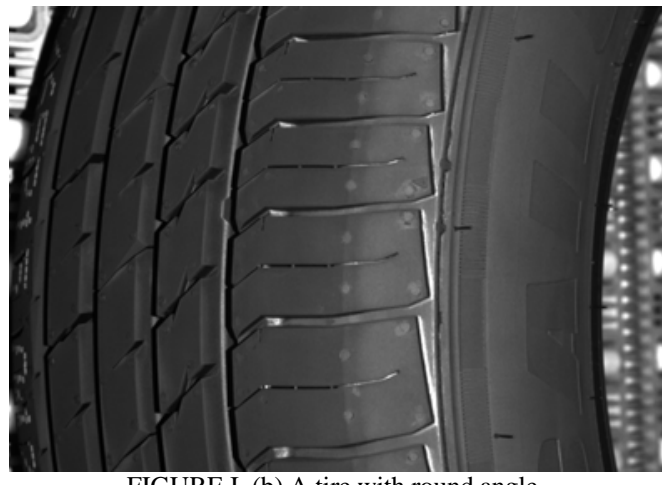

FIGURE I. (b) A tire with round angle.

FIGURE I. TIRES WITH DIFFERENT TIRE ANGLES.

The rest of this paper is organized as follows. Section II describes the proposed framework including filtering, locating tire angle and ridge detection. An evaluation of the proposed framework is presented in Section III. Section IV reaches some conclusions.

\section{PROPOSED FRAMEWORK}

\section{A. Filtering}

The point cloud datasets collected by a 3D point cloud data acquisition camera usually contain some data that does not belong to the tire or has no help for type determination, the part in red dotted circle seen in Figure II. Therefore, it is necessary to carry out a filtering procedure based on coordinate position relationships to improve the efficiency of the subsequent tire angle determination.

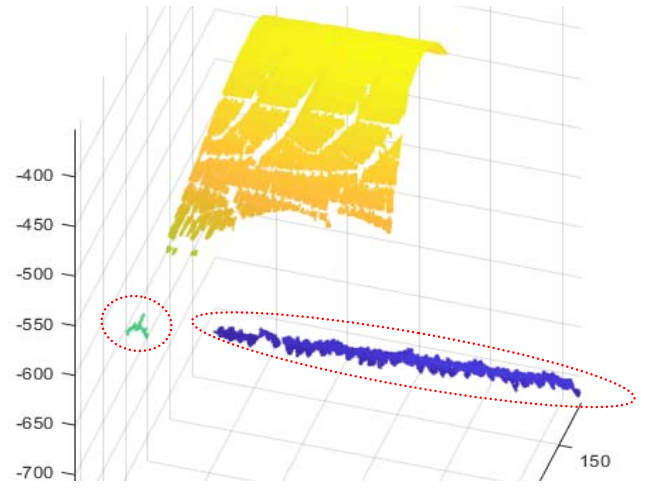

FIGURE II. 3D RECONSTRUCTION OF A TIRE POINT CLOUD DATASET.

\section{B. Locating Tire Angle}

After eliminating the redundant parts, the position of tire angle should be located firstly. In the 3D space, we can find that the position of the tire angle is generally at the corner of the tire surface. Because the tire surface is a curved surface, the position of the corners should be a curve. At the same time, as the tire surface is not smooth, in which there are many dents, the curve is not smooth either. In order to extract tire angle in different position of the tire accurately, we take the median value of $Y$ coordinate in tire point cloud data as the start point $y_{0}$, and pick out some point cloud data whose $Y$ coordinate is within a certain range $\left[y_{0}-\Delta y, y_{0}+\Delta y\right]$, where $\Delta y$ is determined according to the size of the tire point cloud data. In the dataset selected, we cut the data into $X Z$ plane for each $Y$ coordinate whose range is from $y_{0}-\Delta y$ to $y_{0}+\Delta y$, shown in Figure III(a). Then, $2^{*} \Delta y+1$ planes will be obtained, which can also be called images, an example is shown in Figure III(b) denoting by the white parts. According to the size of entire tire point cloud data we collected, the range of $Y$ coordinate is about $120 \mathrm{~mm}$, so the value of $\Delta y$ is usually set to $20 \mathrm{~mm}$, then about $1 / 3$ data is selected for tire angle determination.

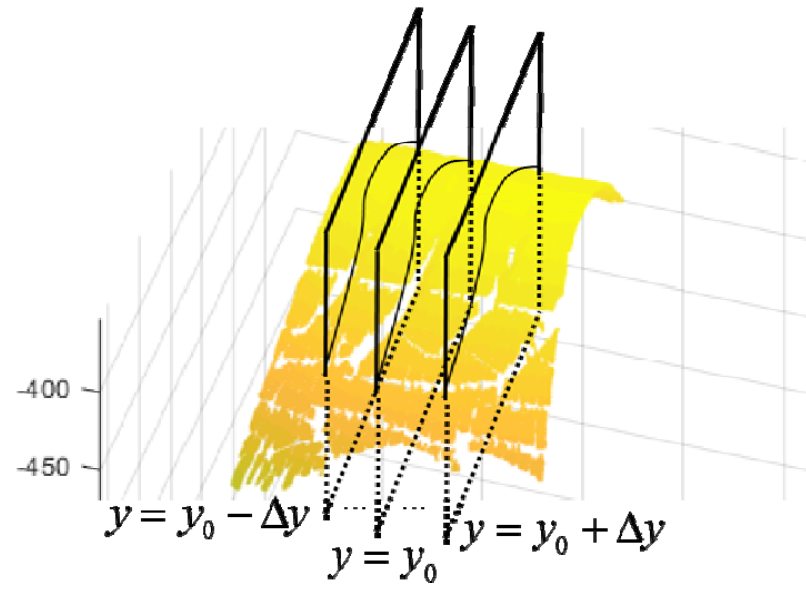

(a)Concrete cut operation

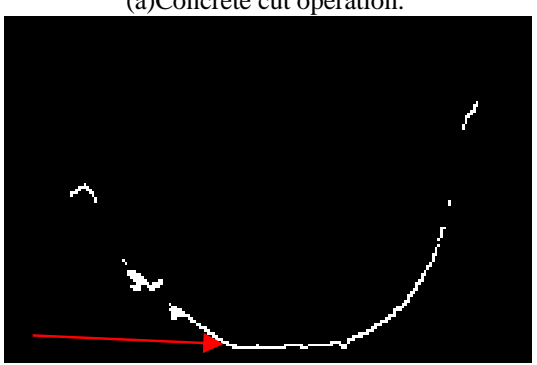

(b) One of the cut planes.

FIGURE III. CUT THE TIRE POINT CLOUD DATA SELECTED ACCORDING TO EACH Y COORDINATE.

In the $2^{*} \Delta y+1$ images, the position of tire angle shown in Figure III(b) the red arrow pointing to always has the following two characteristics: 1) The location of the tire angle is usually located in the lower part of the image. 2) The location of the tire angle is the junction between the front and the side of the tire, and it can be regarded as a corner point in the cut image. So, the location of tire angle determination is changed to corner point detection[7] in the $2 * \Delta y+1$ images. Before applying corner point detection, some preprocessing of images is needed including morphological processing, connected component labeling. The purpose of morphological operation is to make all tire areas in the image become single pixel width for corner detection. And the connected component labeling is to preserve only the region containing the tire angle. Some preprocessing results are shown in Figure IV. Based on the preprocessing results, corner points detection is applied to determine the 
location of tire angle in each image, the red cross shown in Figure IV(b).
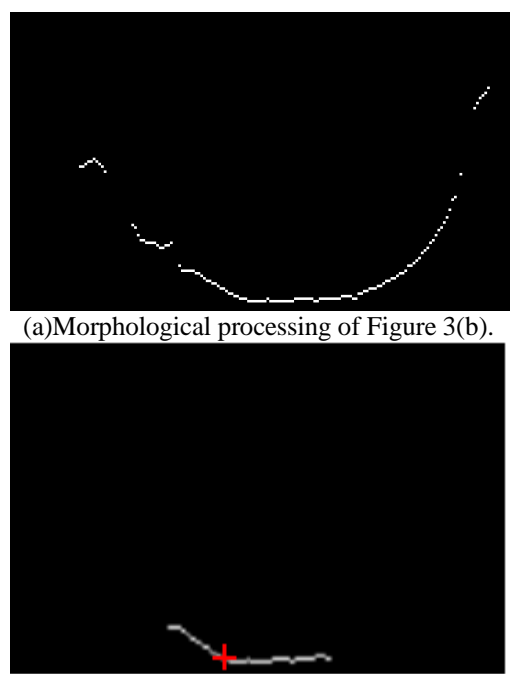

(b) Connected component labeling result of (a)

FIGURE IV. PREPROCESSING RESULTS OF FIGURE III(B).

\section{Ridge Detection}

After locating the position of tire angle, the next step is to determine the sharpness of tire angle. Generally, if the tire angle is sharp, it is equivalent to having a ridge on the position of tire angle. Therefore, whether the tire angle is sharp or round becomes the ridge detection of the tire. If there is a ridge detected, the tire angle is sharp, otherwise it is round. According to [1] and [8], the ridge point in point cloud data is usually the point with extreme curvature value in the main direction. There are two steps in ridge detection:1) surface normal vector estimation;2) principal curvature computation.

For normal estimation, given a geometric surface, it's usually trivial to infer the direction of the normal at a certain point on the surface as the vector perpendicular to the surface in that point. However, since the point cloud datasets that we acquire represent a set of point samples on the real surface, there are two possibilities: 1) obtain the underlying surface from the acquired point cloud dataset, using surface meshing techniques, and then compute the surface normals from the mesh; 2) use approximations to infer the surface normals from the point cloud dataset directly. Though many different normal estimation methods exist, the one that we concentrate on is one of the simplest, and is formulated as follows. The problem of determining the normal to a point on the surface is approximated by the problem of estimating the normal of a plane tangent to the surface, which in turn becomes a leastsquare plane fitting estimation problem. The solution for estimating the surface normal is therefore reduced to an analysis of the eigenvectors and eigenvalues (or PCA Principal Component Analysis) [9] of a covariance matrix created from the nearest neighbors of the query point. More specifically, for each point $p_{i}$, we assemble the covariance matrix $c$ as follows:

$$
c=\frac{1}{k} \sum_{i=1}^{k}\left(p_{i}-\bar{p}\right)\left(p_{i}-\bar{p}\right)^{T}, \quad c \cdot \overrightarrow{v_{j}}=\lambda_{j} \cdot \overrightarrow{v_{j}}, \quad j \in\{0,1,2\}
$$

Where $k$ is the number of point neighbors considered in the neighborhood of $p_{i}, \bar{p}$ represents the 3D centroid of the nearest neighbors, $\lambda_{j}$ is the $j$-th eigenvalue of the covariance matrix, and $\overrightarrow{v_{j}}$ the $j$-th eigenvector.

Since matrix $c$ is symmetric and positive semi-definite, the eigenvalue $\lambda_{j}$ including $\lambda_{0}, \lambda_{1}, \lambda_{2}$ are all real-valued. Supposed that the three eigenvalues meet the equation $\lambda_{0} \leq \lambda_{1} \leq \lambda_{2}$, then we can use the eigenvalue $\overrightarrow{v_{0}}$ of the smallest eigenvalue $\lambda_{0}$ to approximate the surface normal $n_{i}$ at point $p_{i}$ [10]. Meanwhile, in order to keep the normal vector direction consistent, use (2) to adjust all the surface normals.

$$
n_{i} \cdot n_{j}<0(i \neq j)
$$

According to [10] and [11], MLS (moving least squares) surface is used to calculate the curvature, which can quickly and effectively calculate the Gaussian curvature, mean curvature and principal curvature of each point in scattered point clouds at a considerable noise level. The MLS surface is defined as the local minimum of energy function $e(y, a)$ along the direction of vector field $n(x)$.

$$
n(x)=\frac{\sum_{p_{i} \in Q} \overrightarrow{v_{i}} \theta\left(x, p_{i}\right)}{\left\|\sum_{p_{i} \in Q} \overrightarrow{v_{i}} \theta\left(x, p_{i}\right)\right\|}
$$

$$
e(y, a)=\sum_{p_{i} \in Q}\left(\left(y-p_{i}\right)^{T} a\right)^{2} \theta\left(y, p_{i}\right)
$$

Where, $y$ and $a$ are position vector and direction vector respectively, $p_{i}$ is a point in point cloud dataset $Q, \overrightarrow{v_{i}}$ is the normal vector of point $p_{i}$. The minimum of $e(y, a)$ will be obtained by a partial derivative of function (5). Then, the Gaussian curvature and mean curvature of each point will be obtained by (6) where $H(g(x))$ is the Hessian matrix of $g(x)$ and $\operatorname{Det}(A), \operatorname{Trace}(A)$ denote the determinant and the trace of the matrix $A$ correspondingly. What we need is a larger principal curvature $k_{1}$, which is defined in (7).

$$
g(x)=n(x)^{T}\left(\left.\frac{\partial e(y, n(x))}{\partial y}\right|_{y=x}\right)
$$




$$
\left\{\begin{array}{c}
k_{\text {Gaussian }}=-\frac{\operatorname{Det}\left(\begin{array}{l}
H(g(x)) \\
\nabla g(x)
\end{array} \nabla^{T} g(x)\right.}{\left\|\nabla^{T} g(x)\right\|^{4}} \\
k_{\text {Mean }}=-\left(\frac{\nabla g(x) \cdot H(g(x)) \cdot \nabla^{T} g(x)-\|\nabla g(x)\|^{2} \cdot \operatorname{Trace}(H)}{\|\nabla g(x)\|^{3}}\right) \\
k_{1}=k_{\text {Mean }}+\sqrt{k_{\text {Mean }}^{2}-k_{\text {Gaussian }}}
\end{array}\right.
$$

Through exploring the tire point cloud datasets we collected, only the sharp tire angle is usually composed of many ridges whose length more than $10 \mathrm{~mm}$ along $Y$ axis, and the ridges are always composed of many adjacent points who always have $k_{1}$ greater than 0.1 . Therefore, for the sharpness of tire angle determination, we only need to count the number of ridges with $k_{1}$ greater than 0.1 and the length of which is more than $10 \mathrm{~mm}$ along $Y$ axis at the position of the tire angle. Normal vectors of points at the position of tire angle and ridge detection results for a sharp tire angle are shown in Figure V, the part in the red circle denotes the location of tire angle.

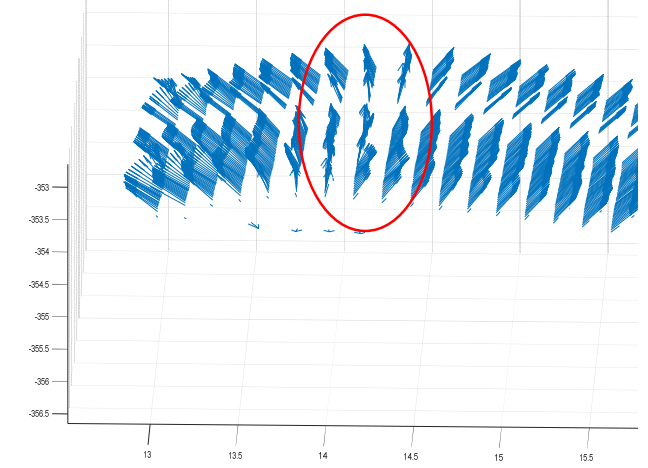

(a)Normal vectors at some positions near the sharp tire angle.

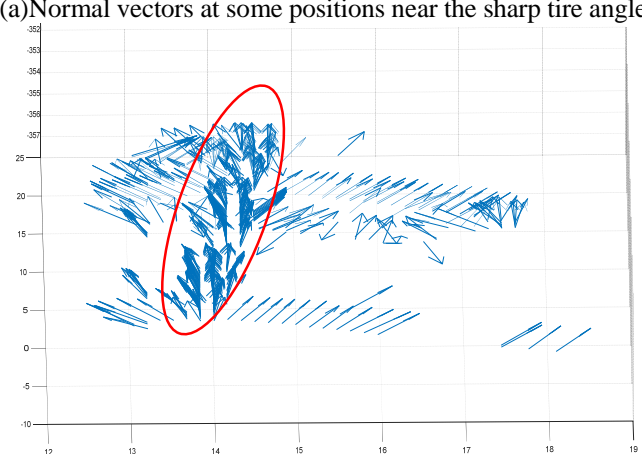

(b) Ridge detection results of (a)

FIGURE V. NORMAL VECTORS AND RIDGE DETECTION OF SHARP TIRE ANGLE.

\section{EVALUATION}

To evaluate the proposed framework, the selected data of this paper are 145 point cloud datasets of tires, in which there are 14 tires with sharp angle and the others with round angle. All experiments are performed on a PC with Win10 OS, 8GB memory, and 256GB SSD (solid state disk) and Matlab R2018a integrated development environments.

In the evaluation experiments, each point cloud dataset has a size of about $13 \mathrm{MB}$, and about 5 seconds are needed for each dataset processing, which can completely meet the efficiency requirement of production line. The sharpness determination results of these 145 datasets are shown in Table 1.

TABLE I. EXPERIMENTAL RESULTS OF 145 POINT CLOUD DATASETS

\begin{tabular}{|c|c|c|}
\hline Type Data & $\begin{array}{c}14 \text { tires with sharp } \\
\text { angle }\end{array}$ & $\begin{array}{c}131 \text { tires with } \\
\text { round angle }\end{array}$ \\
\hline Sharp & 13 & 2 \\
\hline Round & 1 & 129 \\
\hline
\end{tabular}

From Table 1, we can see that 1 tire with sharp angle and 2 tires with round angle have been wrongly classified. Through one step debugging, it is found that the problem mainly lies in the location algorithm of tire angle, who locates a wrong position of tire angle. Generally speaking, the proposed framework has higher accuracy and efficiency for sharpness of tire angle determination and it can be applied in the production line.

\section{CONCLUSIONS}

In this paper, a sharpness of tire angle determination framework is proposed, which contains not only 2D algorithms such as image processing, but also 3D algorithms such as surface normal vector computation. Morphological processing, connected component labeling and corner point detection are used to determine the location of tire angle. Ridge detection including surface normal vector and curvature computation are introduced to determine the sharpness of tire angle. The experimental results show that the proposed framework can efficiently determine the sharpness of tire angle with higher accuracy. Due to if the location of tire angle is wrongly located, it may lead to wrong classification of tire angle, so the followup study will focus on improving the accuracy of the location algorithm of tire angle.

\section{ACKNOWLEDGMENT}

This work is financially supported by the Shandong Provincial Natural Science Foundation of China (Grant No.2016ZRE28057, No. ZR2016DL11). Great thanks to XiaoU Technology Co., Ltd for providing a lot of point cloud datasets of tires with different types.

\section{REFERENCES}

[1] Y.H. Zhang, G.H. Geng, and X.R. Wei, "Valley-ridge feature extraction from point clouds," Optics and Precision Engineering, vol.23, pp.310318, 2015.

[2] S. Gumhold, X.L. Wang, and Maleod, "Feature extraction from poing clouds," Proceedings of the $10^{\text {th }}$ International Meshing Roundtable, pp.293-305, 2001.

[3] M. Pauly, R. Kerser, and M. Gross, "Multi-scale feature extraction on point-sample surfaces,” Computer Graphics Forum, vol.22, pp.281-289, 2003. 
[4] X.F. Pang, M.Y. Pang, and C.X. Xiao, "An algorithm for extrating and enhancing valley-ridge features from points set," Acta Automatica Sinica, vol.32, pp. 1073-1083, 2010.

[5] Q. Merigot, M. Qvajanikov, and L. Guibas, "Voronoi-based curvature and feature estimation from points clouds," Visualization and Computer Graphics, vol.17, pp.743-756, 2011.

[6] Y. An, "Geometric properties estimation and feature identification from 3D point cloud, ” Dalian University of Technology Doctor Dissertation, 2011.

[7] X.C. He and N.H.C. Yung, "Curvature scale space corner detector with adaptive threshold and dynamic region of support," Proceedings of the 17th International Conference on Pattern Recognition, vol. 2, pp/791794, 2004.

[8] T. Judd, F. Durand, and E. Adelson, “Apparen ridges for line drawing," ACM Transcations on Graphics, vol.26, pp.19, 2007.

[9] H. Abdi. and L.J. Williams, "Principal component analysis, ” Wiley Interdisciplinary Reviews: Computational Statistics, vol.2, pp. 433-459, 2010.

[10] Z.L. Ma, M.Q. Zhou, G.H. Geng, J.Z. Sun and J. Li, "Automatic registration algorithm for scattered point clouds based on curvature feature," Applications Research of Computers, vol.32, pp.1878-1880, 1887, 2015.

[11] P.H. Yang and X.P. Qian, "Direct computring of surface curvatures for point-set surfaces,” Eurographics Symposium on Point-Based Graphics, 2007. 